\title{
Neuro-fuzzy Based Maneuver Detection for Collision Avoidance in Road Vehicles
}

\author{
M.A. Zamora-Izquierdo ${ }^{1}$, R. Toledo-Moreo ${ }^{2}$, M. Valdés-Vela $^{1}$, \\ and D. Gil-Galván ${ }^{1}$ \\ ${ }^{1}$ Univ. Murcia, DIIC, Faculty of Computer Science, 30100 Murcia, Spain \\ mzamora@um.es, mdvaldes@um.es \\ http://libra.inf.um.es/ants_vehicles \\ 2 Technical Univ. of Cartagena, DETCP, Edif. Antigones, 30202 Cartagena, Spain \\ rafael.toledo@upct.es
}

\begin{abstract}
The issue of collision avoidance in road vehicles has been investigated from many different points of view. An interesting approach for Road Vehicle Collision Assistance Support Systems (RVCASS) is based on the creation of a scene of the vehicles involved in a potentially conflictive traffic situation. This paper proposes a neuro-fuzzy approach for dynamic classification of the vehicles roles in a scene. For that purpose, different maneuver state models for longitudinal movements of road vehicles have been defined, and a prototype has been equipped with INS (Inertial Navigation Systems) and GPS (Global Positioning System) sensors. Trials with real data show the suitability of the proposed neurofuzzy approach for solving support to the problem under consideration.
\end{abstract}

Keywords: Neuro-Fuzzy, FIS, Intelligent Vehicles, Maneuver Detection, Collision Avoidance.

\section{Introduction}

Intelligent Transportation Systems (ITS) are one of the keys to advance in the development of future societies. ITS-R consists of the applications of telematics to the transportation problem along roads. Navigators, automated vehicles or collision avoidance support systems are a few examples of interesting applications that ITS-R aim [1]. The problem of road vehicles collisions have been intensively reached lately. One interesting approach is based on the situation awareness concept. The issue of situation awareness has been pointed by several authors from the point of view of the artificial intelligence, specially for military purposes [2] - 5. Most of these authors agree to divide multi-sensor data fusion into four levels of increasing situation complexity. Some other approaches, like the one proposed by University of Melbourne, prefer different architecture schemas not necessarily oriented to military scenes [6]. One of the main aspects of situation awareness is the maneuver recognition of the vehicles in a scene. Very different approaches depending on the sensors used can be found in the current literature. Several authors have been focusing their efforts in the recognition of vehicle 
behaviors by using a set of diverse kinematical models. Each model is developed to represent the vehicle behavior in a particular maneuvering state. In [7] a concrete model is selected according to its dynamic state. In 8, FIR filters are used to detect maneuvers and track targets.

The work presented in this paper is based on a Fuzzy Inference System (FIS) for maneuver classification. The navigation system mounts an onboard equipment (OBE) based on GPS/INS, running several extended Kalman filters (EKF) describing the different maneuver states. Previous papers published by the authors showed the suitability of this proposal for road vehicle navigation, even in unfriendly scenarios, like those with low GPS coverage or changing scenarios 9 .

FISs use IF-THEN rules to describe qualitative relationships among variables in the modeled system. Their strength relies on their two fold identity. On the one hand, they look like natural language statements and, therefore, they are suitable for interpretation. On the other hand, they can be regarded as flexible mathematical structures, capable to perform nonlinear mappings between input and output data. Besides, FISs have been widely used to achieve classification tasks [10, given that a fuzzy classifier is only a FIS with crisp and discrete outputs. Rules in a FIS are usually obtained from expert knowledge. However, these kind of models may show poor performance. This can be sorted out by means of methods to build or update fuzzy models from numerical input-output data. This approach is called Data Driven Fuzzy Modeling (DDFM) [11/12. However, they pay little attention to the transparency of the resulting FISs what is their main drawback. Nevertheless, in the last years several approaches to achieve a trade-off between performance and transparency have arisen [13.

In this work we apply DDFM techniques to build a FIS for classifying road vehicles maneuvers. Concretely, it has been applied a clustering technique followed by a neuro-fuzzy approach in which the adaptive capabilities of neural networks have been used to tune the parameters of the target FIS. As we will see in Section 6 . very promising performance results have been obtained. Nevertheless, the current paper must be regarded as a first approximation to the application of fuzzy models to the problem under consideration. Future investigations will be devoted to the generation of more interpretable and accurate FIS for maneuver classification.

\section{System Architecture: Quadrant}

Quadrant is an architecture for ADAS (Advanced Driver Assistance Systems) applications based on the separation of four layers according the level of abstraction of the fusion performed, paying special attention to its communication framework 14. First layer (or sensor layer) is in charge of the measurement collection, sorting and synchronization. These measurements are sent to upper layers via the sensor network. Secondly, fusion layer fuses the data coming from the sensors. This layer, oriented to the interpretation of the vehicle behavior to be performed in the following phase, is described by a set of models, representing different dynamic states of the vehicle. The difficulty to find a unique 
behavior that properly describes all possible maneuvers of a vehicle encourages the definition of multiple models to better describe its movements along roads. Besides, whether the system is able to determine the dynamic behavior of the vehicle, the use of multiple models supplies additional information relative to the maneuver state. Fused data are then supplied to the third layer via ad hoc networks in the vehicle environment. Those networks are supported by WLAN connection availability, thanks to the WIFI PCMCIA card installed in the vehicle computer. Third layer or interpretation layer is in charge of dynamic classification of the vehicle and scene interpretation. The use of the multiple models in the lower layer eases the dynamic classification process, according to the selection of the maneuver states of the vehicle, performed as explained in subsequent Sections. Finally, interpreted data (and any other data coming from previous phases) can be used by the application in order to provide the final service to the user.

A scene will be defined by the information of the vehicle itself, those surrounding, and geographical information of interest in the area (type of road, speed limits, etc.). Each vehicle in the scene is in charge of identifying its maneuver state and reporting it, along with its pose, to the rest of vehicles in the scene (typically a few close enough to be involved and capable to communicate via ad hoc WLAN). On board equipment is described in details in $[9]$.

\section{Multiple Model Filtering}

The basic idea of using multiple models is based on the fact that a vehicle performs very different maneuvers depending on scenario features. For road vehicles, typical maneuvers in highways differ from those usual in city environments. Thus, a single vehicle model can hardly represent all possible maneuvers, and the use of multiple models, representing different maneuver states and running in parallel is advisable. The output of the multiple model approach is typically the model with highest probability value, or a weighted composite of the individual filters [15]. In our proposal we have distinguished between lateral and longitudinal movements of the vehicle. In this paper we focused on longitudinal movements.

\subsection{Vehicle Models}

Most common approach found in the current literature for multiple modeling of longitudinal movements, proposes models representing constant acceleration (CA) and constant velocity (CV) maneuver states. The combination of CV and CA models has been analyzed lately in the literature [16. However, some of these authors found problems with the transition to the CA model, including those who used the IMM method [17. In this paper, the tuning of the CA noise parameters to avoid often unrealistic switches from one state to the other was found problematic, impoverishing the perception of the situation. In case of highways, typical accelerations and decelerations do not last long enough to 
accomplish the transition from the CV state, to the CA state. To overcome these difficulties, we propose two different constant acceleration models with different noise parameter adjustments, and a stationary model to consider non-maneuver state. Kinematical model proposed is a simplified bicycle model, in which the orientation of the acceleration and velocity are assumed to be equal. Different tests done proved that this assumption can be done for highway scenarios.

Acceleration/Deceleration (AD). State vector of the acceleration/deceleration model is $\mathbf{x}_{\mathrm{AD}}=(x, y, \phi, v, \omega, a)$, representing east, north, velocity angle, velocity, yaw rate of turn, and the acceleration, in the center of mass of the vehicle. The dynamics of this model are described by

$$
\begin{aligned}
\dot{\mathbf{x}}_{\mathrm{AD}}= & {\left[\begin{array}{lllllll}
(v+a t) \cos (\phi) & (v+a t) \sin (\phi) & \omega & a & 0 & 0
\end{array}\right]^{\prime}+} \\
& {\left[\begin{array}{llllll}
0 & 0 & 0 & 0 & \eta_{\omega_{\mathrm{AD}}} & \eta_{a_{\mathrm{AD}}}
\end{array}\right]^{\prime} }
\end{aligned}
$$

where $\eta_{\omega_{\mathrm{AD}}}$ and $\eta_{a_{\mathrm{AD}}}$ are white noise terms representing the errors due to model assumptions of constant acceleration and constant yaw rate.

Cruise (CR). State vector and differential equation of the cruise model are the same as in the AD model (1). As previously commented many authors proposed constant velocity models for cruise maneuver state. However, this has been found problematic in several cases. In our approach, both $\mathrm{AD}$ and $\mathrm{CR}$ maneuver states are defined by similar $\mathrm{CA}$ models, defining higher noise values for $\mathrm{AD}$ state $\left(\eta_{\omega_{\mathrm{AD}}}, \eta_{a_{\mathrm{AD}}}\right)$ in order to fulfill higher dynamics.

Stationary (S). In this case, the vector state is simplified being $v=\omega=a=0$, and the differential equation

$$
\dot{\mathbf{x}}_{\mathrm{S}}=\left[\begin{array}{llllll}
\eta_{x_{\mathrm{S}}} & \eta_{y_{\mathrm{S}}} & \eta_{\phi_{\mathrm{S}}} & 0 & 0 & 0
\end{array}\right]^{\prime},
$$

where $\eta_{x_{\mathrm{S}}}, \eta_{y_{\mathrm{S}}}$ and $\eta_{\phi_{\mathrm{S}}}$ are white noise terms representing the errors due to the model assumptions. Noise parameters of the models are tuned starting from the sensor specifications. Observations for the $\mathrm{AD}, \mathrm{CR}$ and $\mathrm{S}$ individual filters are GPS north and east values $\left(x_{\mathrm{gps}}, y_{\mathrm{gps}}\right)$, odometry velocity $\left(v_{\mathrm{odo}}\right)$ and inertial measurements for angular rate $\left(\omega_{\text {ins }}\right)$ and longitudinal acceleration $\left(a_{\text {ins }}\right)$.

\section{TSK Fuzzy Inference System}

In this paper we are concerned with FISs of type TSK [18] (see figure1(a)). In such kind of FISs the rule consequents are usually constant linear functions of the inputs:

$$
\begin{aligned}
& \text { IF } x_{1} \text { is } A_{11} \text { AND } \ldots \text { AND } x_{p} \text { is } A_{1 p} \text { THEN } y=a_{10}+a_{11} x_{1}+\ldots+a_{1 p} x_{p} \\
& \text { IF } x_{1} \text { is } A_{21} \text { AND } \ldots \text { AND } x_{p} \text { is } A_{2 p} \text { THEN } y=a_{20}+a_{21} x_{1}+\ldots+a_{2 p} x_{p} \\
& \ldots \\
& \text { IF } x_{1} \text { is } A_{r 1} \text { AND } \ldots \text { AND } x_{p} \text { is } A_{r p} \text { THEN } y=a_{r 0}+a_{r 1} x_{1}+\ldots+a_{r p} x_{p}
\end{aligned}
$$

where $r$ is the number of rules, $p$ is the number of input variables, $x_{1}, x_{2}, \ldots, x_{p}$ are the input variables and $y$ is the output one. $A_{i 1}, A_{i 2}, \ldots, A_{i p}$ are the antecedent fuzzy sets of $i$-th rule. They can have an attached linguistic label. The 


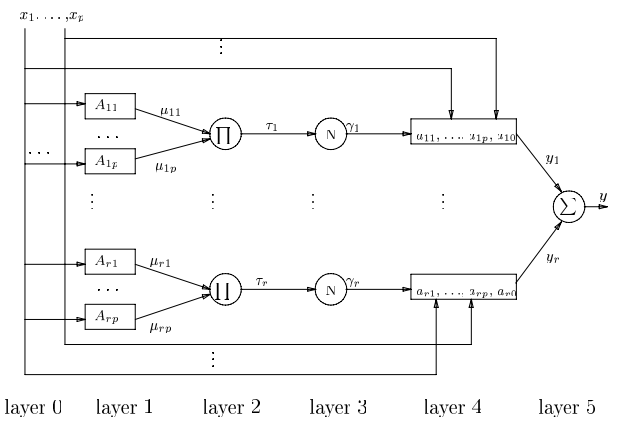

(a)
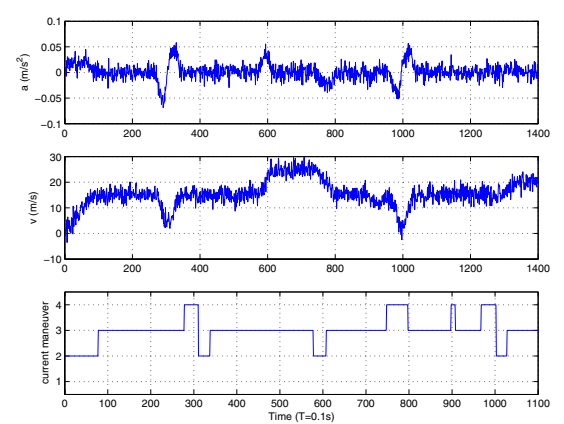

(b)

Fig. 1. (a) ANFIS architecture. (b) Training data coming from circuit 4.

fuzzy sets serve as a smooth interface between the numerical nature of input and output variables and linguistic labels.

A type of TSK FIS where every consequent is a crisp value is considered a special case of TSK FIS with zero-order polynomials in the consequents, the so-called Zero-Order TSK FIS. This is more suitable for classification tasks. As we will see in our maneuver classification problem a natural number from 1 to 4 is associated to each maneuver. Nevertheless, first-order TSK FISs (the ones with linear consequents) can also be used.

To obtain the output inferred with a TSK model (of any order), first of all it is necessary to compute the degree of fulfilment of the $i$ th rule, denoted as $\tau_{i}$, with $i=1, \ldots, r$. This degree of fulfillment is given by the true value of the proposition " $x_{1}$ is $A_{i 1}$ AND ... AND $x_{p}$ is $A_{i p}$ ", the meaning of which is given through a t-norm as, for instance, the product operator. Therefore, given the input $x_{1}, \ldots, x_{p}$, the degree of fulfillment of the $i$-th rule is computed as:

$$
\tau_{i}=A_{i 1}\left(x_{1}\right) \cdot A_{i 2}\left(x_{2}\right) \cdot A_{i p}\left(x_{p}\right)
$$

Finally, FIS output $y$ is obtained adding partial outputs of each rule, weighted with their respective degrees of fulfillment through the equation:

$$
y=\frac{\sum_{i=1}^{r} \tau_{i} y_{i}}{\sum_{i=1}^{r} \tau_{i}}
$$

where $y_{i}$ is the output of the $i$-th rule:

$$
\begin{array}{ll}
y_{i}=a_{i 0}+a_{i 1} x_{1}+\ldots+a_{i p} x_{p}, & \text { in a first-order TSK } \\
y_{i}=a_{i 0}, & \text { in a zero-order TSK }
\end{array}
$$

Our strategy starts with the selection of the number of rules. Instead of an automatic selection, a suitable number can be chosen according to the distribution of the available input-output data. Therefore, this number is an initial parameter to a clustering optimization method resulting in a first approximation to the 
target FIS. Finally, a neuro-fuzzy architecture is fed with the centroids detected. Afterwards, the adaptive capabilities of this network are used in order to adjust FIS parameters.

\section{A FIS Maneuver Classification: DDFM Process}

In general, three main stages compose a DDFM process: first, the number of rules must be detected (rules number identification). After that, a rough approximation to the set of rules is obtained (rules generation). Afterward the fuzzy sets involved in the rules are adjusted in the parameter optimization stage to better mimic the system behavior.

In this work, instead of the automated selection, a suitable number is chosen manually according to the visual distribution of the available input-output data. Therefore this number is an initial parameter to a subsequent clustering optimization method applied to the input-output data. Every cluster can be regarded as a rough fuzzy rule. Hence, their centroids are initial values to the fuzzy sets involved in the FIS which will be tuned using the adaptive capabilities of the neuro-fuzzy architecture.

\subsection{Rules Generation}

Fuzzy C-Means Clustering Method (FCM) [19] is one of the most used methods for optimization of an initial partition of data. Given a set of examples, the algorithm must be provided with a $c$ number a centroids (set beforehand) and an initial fuzzy $c$-partition of the data. Then, algorithm optimizes the $c$-partition in such a way that certain cost function $J$ is minimized. This function is the weighted within groups sum of squared-errors.

At each step, three operations are successively carried out until the centroids are stable with respect to a given tolerance $(\epsilon)$ : (1) computation of the centroids (assuming that the membership degrees composing the partition are constants numbers); (2) calculus of the distances from each datum to every centroid (the most commonly used measurement for distance is Euclidean, inducing spherical clusters); (3) update the membership degree of each data point to every cluster (assuming that the centroids remain constant).

The partition of the input-output data obtained by means of this clustering method is considered as first approximation to the target FIS . These clusters centroids are the initial parameters for the fuzzy sets involved in the FIS. Next step is the tuning of those parameters, described in the next Section.

\subsection{Parameter Optimization}

In order to optimize the parameters, we use a neuro-fuzzy approach. Neuro-fuzzy mechanisms merge the adaptive capabilities of artificial neural networks with the human-readable information representation provided by FISs. Concretely we use the Adaptive Neuro-fuzzy Inference System (ANFIS) 20] that is functionally 
equivalent to a TSK FIS. It is compound of five layers. Nodes in layer 1 and layer 4 correspond to the antecedent and consequent parameters of the TSK FIS respectively. The ANFIS architecture is shown in the Fig. 1(a).

We have assumed fuzzy sets with gaussian membership functions. Therefore, the mean of the fuzzy sets in the antecedent layers are initialized with the centroids obtained in the previous phase of DDFM. Next, training is carried out during several epoches (iterations). Every epoch of the training is compound of a forward and backward pass. In the forward pass the network is evaluated for every input datum and the rule consequent parameters are identified by means of the Least-Squares Estimator. Afterwards, errors for every training datum are calculated and, in the backward pass, error signals are propagated and the rule antecedents modified through backpropagation.

\section{Experimental Tests and Results}

In order to train and test the proposed system, a prototype vehicle equipped with two IMU (Inertial Measurement Unit) sensors (MT9-B Xsens and Xbow VG-400 IMU units) based on low cost MEM (Micro-Electro-Mechanical) technology has been used. A Trimble DGPS sensor and the odometry of the car were also utilized to obtain the data set. With this information, sensor fusion layer estimates the vehicle pose through different extended Kalman filters, each one for one kinematic model. Real data coming from five different circuits (6908 instances) have been used in the experiments.

Different sets of input data were analyzed, deciding finally to use four inputs to feed the target FIS: two last velocity measurements $(v(k)$ and $v(k-1))$ provided by the odometry of the car, longitudinal acceleration $\left(a_{x}(k)\right)$ provided by the IMU unit, and previous output $(o(k-1))$. In these experiments, no angular information is taken into account since we focus exclusively on longitudinal maneuvers. Concretely, four different maneuver states have been considered: stationary (denoted as ST and represented by constant value 1 in a zero-order $\mathrm{TSK})$, acceleration $(\mathrm{AC} / 2)$, cruise $(\mathrm{CR} / 3)$ and deceleration $(\mathrm{DC} / 4)$. Despite the fact that from the point of view of kinematics, acceleration and deceleration can be described by a single model, we make this distinction to check the DDFM process performance for this case.

Input-output data coming from four of the five mentioned circuits were used for training. Fig. 1(b) shows circuit 4 acceleration and velocity measurements, and manual maneuver labeling. Given the data distribution in the input-output space, we decided to set $c=12$ in the FCM algoritm, that is, 12 clusters and hence 12 rules. The tolerance index $\epsilon$ was set to $10^{-4}$. Let us call FCS the model of 12 clusters generated by means of the FCM method. Each cluster was projected into each one of the axis obtaining a first approximation of the target FIS (let us call it FCS-p). Afterwards, the ANFIS architecture was fed twice with this rough FIS in order to obtain, respectively, zero and first order TSK FIS. After training twice for 100 epoches, two different classifiers were obtained, TSK-0 and TSK-1. 
Final and intermediate FISs were validated using the circuit 5. In Table 1(a) training and validation errors for every FIS are deployed, where RMSE stands for Root Mean Squared Error (RMSE) calculated as

$$
R M S E=\sqrt{\frac{\sum_{i=1}^{n}\left(y_{i}^{t}-\hat{y}_{i}^{t}\right)^{2}}{n}}
$$

being $y_{i}^{t}$ and $\hat{y}_{i}^{t}$ inferred and real outputs for the $i$-th datum, with $n$ number of data. It must be remarked that training and validation errors were comparable, indicating the absence of overtraining.

It can be observed that TSK-1 presents better performance than TSK-0 due to the more powerful approximation capabilities of the polynomial consequents. On the other side, TSK-0 is more transparent since most of the rule consequents match the maneuver themselves (unfortunately, it cannot be shown due to space limitations). The confusion matrixes of TSK-0 and TSK-1 classifiers are shown in Tables 1(b) and 1(c) respectively, where each column of the matrix represents instances in a inferred maneuver, while each row represents instances in reference maneuver state. Both models classify noticeably well stationary instances while TSK-1 is better than TSK-0 classifying other maneuver states.

Table 1. : (a) RMSE of different FISs. (b) TSK-0 confusion matrix. (c) TSK-1 confusion matrix.

(a)

\begin{tabular}{|c||c|c|}
\hline FIS & RMSE Tr. & RMSE Va. \\
\hline \hline FCS & 0.47 & 0.83 \\
\hline FCS-p & 0.40 & 0.54 \\
\hline TSK-0 & 0.24 & $\mathbf{0 . 2 8}$ \\
\hline TSK-1 & 0.15 & $\mathbf{0 . 2 4}$ \\
\hline
\end{tabular}

(b)

\begin{tabular}{|c|c|c|c|c|c|c|c|c|c|}
\hline & ST & $\mathbf{A C}$ & $\mathrm{CR}$ & $\mathbf{D C}$ & & ST & $\mathrm{AC}$ & CR & DC \\
\hline$\overline{\mathbf{S T}}$ & 499 & 28 & 0 & 0 & $\mathbf{S T}$ & 499 & 1 & 0 & 1 \\
\hline $\mathbf{A C}$ & 0 & 231 & 3 & 1 & $\overline{\mathrm{AC}}$ & 1 & 306 & 6 & \\
\hline CR & 0 & 50 & 857 & 6 & CR & 0 & 3 & 854 & \\
\hline$\overline{\mathrm{DC}}$ & 2 & $\overline{12}$ & 0 & 409 & $\overline{\mathrm{DC}}$ & 1 & 11 & 0 & 40 \\
\hline
\end{tabular}

In Fig. 2(a) data extracted from the validation circuit are deployed. Fig. 2(b) shows the maneuver inferred by TSK-0 and TSK-1 classifiers versus estimated truth adjusted by manual labeling. It can be observed, for example, that many of errors are concentrated around instant $\mathrm{t}=200$ tenths of s., where AC points are classified as CR in TSK-1 and DC in TSK-0 due to the large oscillations in $v(t)$.

Once a fuzzy classifier has been obtained, it can be used by the algorithm. In a higher level the system decides which kinematic model will be used, taking into account the output of the fuzzy classifier. With the mentioned four inputs, the system is capable to learn about changes in the velocity fusing information coming from two different sensors. Last output adds information of the current maneuver to the system. 
(a)
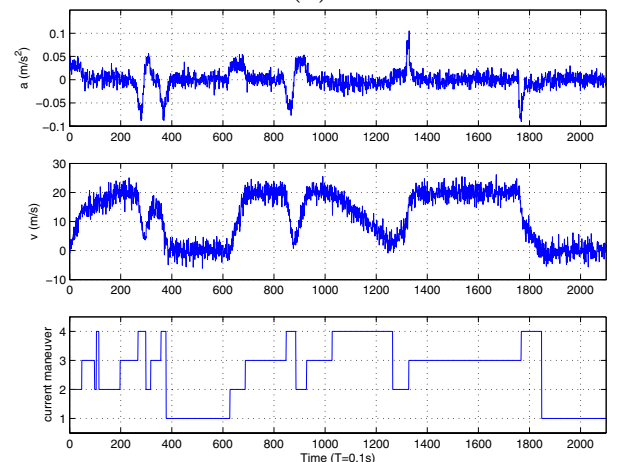

(b)
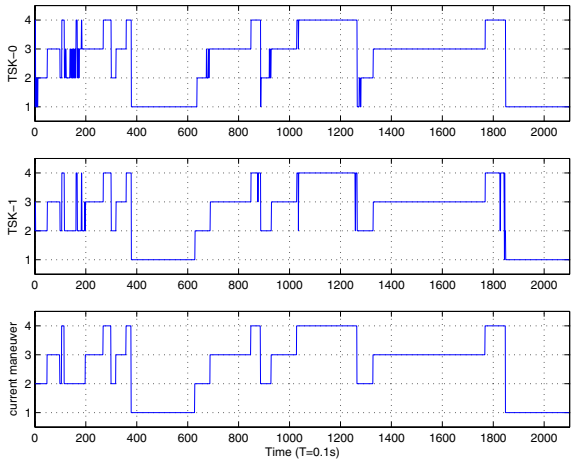

Fig. 2. (a) Circuit 5 used for evaluation. (b) Reference maneuvers and those inferred by TSK- 1 and TSK-0 FISs.

\section{Conclusions and Future Work}

The use of a model-set representing different state maneuvers of a road vehicle provides an appropriate modeling of the vehicle behavior in many different dynamic situations with realistic pose and noise estimates. This paper proposes a fuzzy classifier for state maneuver detection that has been obtained from inputoutput data by means of a clustering technique and a subsequent neuro-fuzzy approach.

In this first work, promising results have been obtained. Trials performed with real data in a set of road scenarios show the suitability of the proposed system for the problem of detecting different maneuver states for longitudinal movements. Next step is to analyze the system capability for transversal maneuvers, including lane change. Regarding the fuzzy inference system, several improvements can be introduced. In the current experiments the number of fuzzy rules have been fixed a priori. Future works will be focused on the use of automatic methods for the detection of this number directly from data. Further researches will lead us to the generation of more accurate and user-friendly fuzzy rules susceptible of being contrasted with a human expert.

\section{Acknowledgments}

The Authors would like to thank the Spanish Ministerio de Fomento and the European Space Agency (ESA) for sponsoring the research activities under the grants FOM/3929/2005 and GIROADS 332599 respectively.

\section{References}

1. Skarmeta A., Toledo R., Zamora M.A., Ubeda B., Santa J., Sotomayor C.: Master Plan for ITS in Spain. Ministerio de Fomento Español (2006) 
2. Kokar M.M. ,Matheus C.J., Letkowski J.A.: Association in Level 2 fusion, SPIE (2004).

3. Matheus C.J., Kokar M.M., Baclawski K.: A Core Ontology for Situation Awareness. Proceedings of Sixth International Conference on Information Fusion, Cairns, Australia, July (2003), pp. 545-552.

4. Ceruti M.G. and Kamel M.N.: Preprocessing and Integration of Data from Multiple Sources for Knowledge Discovery. International Journal on Artificial Intelligence Tools, vol. 8, no. 3, June (1999), pp. 159-177.

5. Waltz E. and Llinas J.: Multisensor Data Fusion. Artech House, Boston (1990).

6. Dance S., Caelli T., Liu Z.Q.: An architecture for a traffic scene interpretation system. Technical Report 94/12, Dept. of Computer Science. University of Melbourne. (1994).

7. Weiss K., Stueker D., Kirchner A.: Target Modelling and Dynamic Classification for Adaptive Sensor Data Fusion. Proceedings of the IEEE Intelligent Vehicles Symposium 2003 June (2003), pp. 132-137.

8. Hwan Park S., Hyun Kwon W., Oh-Kyu Kwon and P. So Kim: Maneuver Detection and Target Tracking Using State-Space Optimal FIR Filters. Proceedings of the American Control Conference. San Diego, California June (1999). pp. 4253-4257.

9. Toledo R., Zamora M.A. and Gomez-Skarmeta A.F.: A Novel Design of a High Integrity Low Cost Navigation Unit for Road Vehicle Applications, Proceedings of the IEEE-IV 2006, Tokyo, Japan, June (2006) pp.577-582.

10. Gómez-Skarmeta A.F., Valdés M., Jiménez F. and Marín-Blázquez J.G.: Approximative fuzzy rules approaches for classification with hybrid-GA techniques. Information Sciences (2001) vol. 136, no. 1-4, pp.193-214.

11. Babuška R.: Fuzzy Modeling for Control. Kluwer Academic Publishes (1998).

12. Valdés M. Gómez-Skarmeta A.F. and Botía J. A.: Towards a Framework for the Specification of Hybrid Fuzzy Modeling. International Journal of Intelligent Systems (2005), vol 20 no. 2, pp.225-252.

13. Teng Y. W. and Wang W. J., Constructing a User Friendly GA-Based Fuzzy System Directly From Numerical Data. IEEE Transactions on Systems, Man and Cybernetics-Part B (2004), vol.34, no. 5 pp.2060-2070.

14. Toledo R., Sotomayor C. Gomez-Skarmeta A.F., Quadrant: An Architecture Design for Intelligent Vehicle Services in Road Scenarios. Monograph on Advances in Transport Systems Telematics (2006) pp.451-460.

15. Bar-Shalom,Y., and X.R.Li, Multitarget-multisensor tracking: principles and techniques. Storrs, CT. YBS Publishing, (1995).

16. Barrios C., Himberg H., Motai Y., Sadek A.: Multiple Model Framework of Adaptive Extended Kalman Filtering for Predicting Vehicle Location. Proceedings of the IEEE-ITSC 2006, Toronto, Canada, September 17-20, (2006). pp. 1053-1059.

17. Hoffmann C., and Dang T.: Cheap Joint Probabilistic Data Association Filters in an Interacting Multiple Model Design. Proceedings of the 2006 IEEE-MFI 2006. September 3-6, (2006), Heidelberg, Germany. pp. 197-202.

18. Takagi T. and Sugeno M.: Fuzzy Identification of Systems and its Applications to Modeling and Control. IEEE Transactions on Systems, Man and Cybernetic (1985), vol. 15, pp.116-132.

19. Bezdeck J. C.: Pattern Recognition with Fuzzy Objective Function Algorithms. Plenum, New York,(1981).

20. Jang J. S. ANFIS: Adaptive-network-based fuzzy inference systems, IEEE Transactions on Systems, Man, and Cybernetics (1993), vol. 23, no. 3,pps665-685. 